Geheimräte gegen Geheimbünde 
W. Daniel Wilson

\section{Geheimräte gegen Geheimbünde}

Ein unbekanntes Kapitel der klassisch-romantischen Geschichte Weimars

J. B. Metzlersche Verlagsbuchhandlung Stuttgart 


\section{Die Deutsche Bibliothek - CIP-Einheitsaufnahme}

\section{Wilson, W. Daniel:}

Geheimräte gegen Geheimbünde : ein unbekanntes Kapitel der klassisch-romantischen Geschichte Weimars /

W. Daniel Wilson. - Stuttgart : Metzler, 1991

ISBN 978-3-476-00778-0

ISBN 978-3-476-03376-5 (eBook)

DOI $10.1007 / 978-3-476-03376-5$

Dieses Werk einschließlich aller seiner Teile ist urheberrechtlich geschützt. Jede Verwertung außerhalb der engen Grenzen des Urheberrechtsgesetzes ist ohne Zustimmung des Verlages unzulässig und strafbar. Das gilt insbesondere für Vervielfältigungen, Übersetzungen, Mikroverfilmungen und die Einspeicherung und Verarbeitung in elektronischen Systemen.

(C) 1991 Springer-Verlag GmbH Deutschland Ursprünglich erschienen bei J.B. Metzlersche Verlagsbuchhandlung und Carl Ernst Poeschel Verlag GmbH in Stuttgart 1991 


\section{Inhalt}

Vorwort

1 Einleitung. Die Illuminaten und die Illusionen des aufgeklärten Absolutismus

2 Die Verschwörungslegende. Deutsche Illuminaten und Französische Revolutionäre

3 Die Weimarer Illuminaten Goethe und Herzog Carl August

3.1 Die \Schwedenkiste und die Goetheforschung 50

3.2 Die freimaurerische Strikte Observanz und der Beitritt der Weimarer zum Illuminatenorden 55

3.3 Die beginnenden Spannungen zwischen dem Orden und seinen herrschaftlichen Mitgliedern

3.4 Der Streit zwischen Knigge und Weishaupt und Knigges Plädoyer vor den Weimarer Illuminaten (1784) ....................... 86

3.5 Der Streit um die fürstlichen Mitglieder 93

3.6 Die verhinderte Berufung Weishaupts nach Jena (1785) .... 106

3.7 Zusammenfassung 130

4 Goethes und Carl Augusts Unterdrückung der Geheimbünde in Jena und die Illuminatenthematik in Goethes Werken 
5 Opfer und Vertreter der Verschwörungslegende in Weimar und Jena im Zeitalter der Französischen Revolution .............................. 163

5.1 Wielands Aufnahme der Verschwörungslegende in den Teutschen Merkur (Mit einem Exkurs zu Schiller 163

5.2 Herders Illuminatentätigkeit und seine politische $>$ Wende .189

5.3 Fichte, F. Schlegel und andere Jenaer unter dem Druck der Reaktionäre 212

6 Schluß: Subjektfiktion und Machtverhältnisse 256

DOKUMENTE

1. Stolberg-Roßla an Bode, 28.5.1783 (Auszug)............................2269

2. Bode an Knigge, 12.6.1783 (Auszug)............................................ 269

3. Bode an Unbekannt, Kopie, 21.6.1783 (Auszug)...................... 271

4. Herzog Carl August von Weimar, "Pro Voto«, [vor

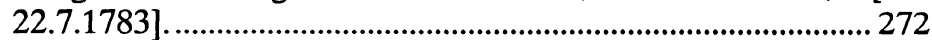

5. Weishaupt an Bode, 29.7.1783 (Auszug)................................. 274

6. Herzog Ernst II. von Gotha an Bode, 10.8.1783 (Auszug).... 277

7. Georg Laurentius Batsch an Bode, 12.8.1783........................... 278

8. Bode an Herzog Ernst II. von Gotha, »Unterthänigstes P[ro] M[emoria]«, 18.8.1783 (Auszug).

9. Joachim Peter Tamm an Bode, 30.9.1783 (Auszug)................. 279

10. Bode an Herzog Ernst II. von Gotha, 22.10.1783 (Auszug). .. 280

11. Goethe an Bode, [vor 26.10.1783]............................................. 282

12. Knigge an Bode, 14.11.1783 (Auszug)...................................... 283

13. Musäus an Bode, 28.12.1783 (Auszug). ..................................... 283

14. Graf von Stolberg-Roßla an Bode, 3.1.1784 (Auszug)............. 284

15. Bode an Herzog Ernst II. von Gotha, 11.1.1784 (Auszug)..... 284

16. Bode an Herzog Ernst II. von Gotha, 19.1.1784 (Auszug)..... 285

17. Bodes Notizen über Knigges Besuch, 10.-13.2.1784 (Aus-

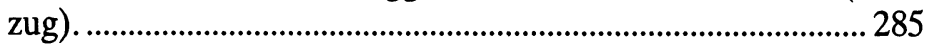

18. [Bode,] »Actum«, 12.2.1784; Knigge, »Nachtrag «, 29.2.1784. 287

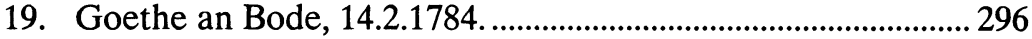

20. Goethe an Bode, [etwa Anfang April 1784] ............................... 297

21. Herzog Ernst II. von Gotha an Bode, 2.5.1784 (Auszug)....... 298

22. Bode an Herzog Ernst II. von Gotha, 3.5.1784 (Auszug)....... 299 
58. Herzog Carl August von Weimar an Christian Gottlob Voigt,

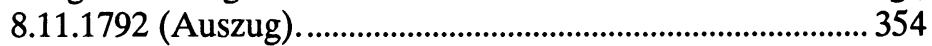

59. Herzog Carl August von Weimar an Christian Gottlob Voigt, 24.11.1792 (Auszug) 355

Anhang 1: Die Weimarer, Jenaer und Gothaer Illuminaten 357

Anhang 2: Die sSchwedenkiste، 363

Anhang 3: Der Ordenskalender .365

Abkürzungen 366

Bibliographie 369

Personenregister 381 


\section{Vorwort}

Für Außenstehende ist die an Besessenheit grenzende Beschäftigung der Deutschen mit Goethe ein faszinierendes Phänomen, denn in anderen westlichen Kulturen findet man kaum eine ähnliche Belastung der nationalen Identität durch eine übermächtige literarische Persönlichkeit. Wenn Deutsche gefragt werden, was in der deutschen Vergangenheit Großes geleistet worden sei, so weisen sie verständlicherweise meist nicht auf politische, sondern auf kulturelle Figuren hin, allen voran Goethe. Schon an diesem Umstand wird deutlich, daß hinter dieser Dominanz auch ein politisches Programm steckt, aber in der öffentlichen Diskussion wird allzu wenig über die resultierenden Verzerrungen nachgedacht. Daß Goethe besonders in der DDR das kulturelle Bewußtsein dominieren und mit politisch progressiven Helden in einer Reihe stehen konnte, ist aus der Kulturpolitik der DDR leicht zu verstehen; aber auch im Westen sind ähnliche Urteile zu konstatieren. Nach der »Stunde Null« des Jahres 1945 war, so Karl Robert Mandelkow, in der BRD sowie in der DDR »der Rückbezug auf Goethe Bestandteil der Suche nach einer neuen nationalen Identität, in beiden Lagern, so unterschiedlich die Voraussetzungen und Traditionen der Rezeption auch waren, griff die Berufung auf Goethe weit über die literarisch-künstlerische Bedeutung seines Werkes hinaus, sie wurde zur jeweils unterschiedlich interpretierten - Anknüpfung an die wahre, die eigentliche, die humanistische Tradition der Deutschen, die der Welt durch die jüngste faschistische Vergangenheit verdeckt worden war. Goethe erhielt Alibifunktion, er wurde eines der vornehmlichen Vehikel der Wiederherstellung des beschädigten Selbstbewußtseins der Deutschen in beiden deutschen Staaten ${ }^{1}{ }^{1}$ Auch in der DDR versuchte man also, den Geheimrat Goethe in den Dienst einer progressiven Tradition zu stellen; ein Gang durch das Goethe-Haus in Weimar konfrontiert den Besucher mit fast jedem halbwegs fortschrittlichen Satz, den Goethe in über sechs Jahrzehnten geschrieben hat. Dabei mußte man natürlich die an sich bekannte Tatsache geflissentlich übersehen -

1 Mandelkow: Goethe in Deutschland 1: $9 \mathrm{f}$. - Alle Quellen und wissenschaftlichen Untersuchungen werden mit sinngemässen Kurztiteln zitiert; die vollständigen Angaben befinden sich in der Bibliographie. 
in der DDR-Forschung allerdings begannen da Spannungen sichtbar zu werden -, daß Goethe im Grunde konservativ war, ja in vieler Hinsicht reaktionär. Und in diesem Zusammenhang spielten und spielen für den durchschnittlichen Leser von Goethes Werken die amtliche Tätigkeit Goethes sowie die dieser zugrundeliegende Bejahung des absolutistischen Systems in ihrer saufgeklärten Variante gewiß eine sehr geringe Rolle, wenn sie überhaupt zur Kenntnis genommen wurden. So pilgerten 1919 die Gründer der zweiten Republik auf deutschem Boden nach Weimar, um dort unbekümmert den Geist desjenigen Klassikers zu beschwören, der 1793 bei der militärischen Belagerung der ersten Republik auf deutschem Boden in Mainz nicht zufällig hinter den Kanonen der monarchistischen Reaktion dem Bombardement zuschaute.

Auch die Wissenschaft hat die Widersprüche der politischen Goethe- und Klassik-Rezeption nur in Ansätzen gewürdigt. Eine wissenschaftliche Untersuchung über Goethes Verhältnis zum Modell des raufgeklärten` Absolutismus, die zugleich die Bedeutung dieser Bejahung eines starken obrigkeitlichen Staates für die deutsche $\mathrm{Ge}$ schichte zu berücksichtigen und im übrigen auch Goethes gelegentliche Vorbehalte und Zweifel gegenüber diesem Modell detailliert zu analysieren hätte, steht leider noch aus. Goethe hat als Greis seine Meinung zum Phänomen eines durch >Aufklärung gemäßigten Absolutismus gegenüber Eckermann geäußert, eine Meinung, die sich seit seiner Jugend nachweislich kaum geändert hatte: »Nun heißt es wieder, ich sei ein Fürstendiener, ich sei ein Fürstenknecht. - Als ob damit etwas gesagt wäre! - Diene ich denn etwa einem Tyrannen? einem Despoten? - Diene ich denn etwa einem Solchen, der auf Kosten des Volkes nur seinen eigenen Lüsten lebt? - Solche Fürsten und solche Zeiten liegen gottlob längst hinter uns. Ich bin dem Großherzog seit einem halben Jahrhundert auf das innigste verbunden und habe ein halbes Jahrhundert mit ihm gestrebt und gearbeitet; aber lügen müßte ich, wenn ich sagen wollte, ich wüßte einen einzigen Tag, wo der Großherzog nicht daran gedacht hätte, etwas zu tun und auszuführen, das dem Lande zum Wohl gereichte und das geeignet wäre, den Zustand des Einzelnen zu verbessern. « ${ }^{2}$ Diese typische Apologie des raufgeklärten< Absolutismus birgt viele der für dieses politische Modell

2 27.4.1825, MA 19: 519 (vgl. das Verzeichnis der Abkürzungen für alle Siglen). Goethes Werke werden nach der vorzüglichen neuen \Münchner Ausgabe` zitiert, die Briefe womöglich nach der Auswahl in der Hamburger Briefausgabe (HAB), weil dort in einigen Fällen Korrekturen gegenüber der Weimarer Ausgabe (WA) vorgenommen wurden, sonst nach dieser. 
charakterischen Widersprüche; indem Goethe hier alle Fürsten seiner Tage als aufgeklärt hinstellt, verfälscht er nicht nur die Geschichte, indem er den akzidentellen Charakter eines saufgeklärten` Fürsten unterschlägt und die >Despoten< seiner Tage übergeht, sondern er liefert auch künftigen Herrschern - die es nach dem hier implizierten geschichtsphilosophischen Modell nicht mehr geben wird - eine bequeme Legitimation für alles, was sie tun, solange es aus ihrer Perspektive dem Landeswohl dient. Und Goethe übersieht geflissentlich den Fall, wo das Wohl des Landes in Konflikt geraten könnte mit den dynastischen und staatlichen Interessen; denn der aufgeklärte Absolutismus blieb eben durch und durch Absolutismus. Er kann - darin ist wiederum der DDR-Historiographie zuzustimmen, ${ }^{3}$ sowenig sie diese Ergebnisse auf Goethe angewendet hat - als Versuch des absolutistischen Staates verstanden werden, die Aufklärung zu absorbieren, die aufgeklärte Intelligenz also zu vereinnahmen und für oppositionelle Zwecke unschädlich zu machen. Der Fürst des >aufgeklärten`Absolutismus »versuchte, möglichst >aufgeklärt $<\mathrm{zu}$ erscheinen, blieb sich jedoch in seinem wesentlichen Sein gleich $\ll, 4$ also an feudalabsolutistische Interessen gebunden. Die Intelligenz kam diesem Bestreben ihrerseits entgegen: "In Deutschland gewann die Aufklärung [...] ihren ganz spezifischen Charakter durch ihr Bündnis mit dem Absolutismus [...]«.5 Indem die Fürsten auf aufgeklärten Reformkurs umzulenken schienen und vor allem die Intelligenzschicht oberflächlich förderten, entzogen sie einem möglichen antifeudalen Umsturz ${ }^{6}$ die Stoßkraft. Die Intelligenz arran-

${ }^{3}$ Stellvertretend seien hier genannt: Mittenzwei: Über das Problem des aufgeklärten Absolutismus; Schilfert: Deutschland von 1648 bis 1789; auch in der früheren französischen Forschung sind ähnliche Erkenntnisse zu konstatieren, etwa bei Lefebvre: Der aufgeklärte Despotismus, der die provozierende Formulierung verfaßte: "In Wirklichkeit bedeuteten diese humanitären Einrichtungen den Herrschern nichts als geistreiche Spielerei. In der hauptsächlichen Sorge um die Ausweitung ihrer Macht erwogen sie sorgfältig, was sie zum Vorteil ihrer Bestrebungen nutzen konnten und was sie besser fallenlassen sollten « (S. 83); dagegen wenden sich Aretin: Der Aufgeklärte Absolutismus, S. 12, und Walder: Aufgeklärter Absolutismus und Revolution, S. 107. Aretin nähert sich jedoch der Position Lefebvres an: Es sei klar, »daß jeder Herrscher, der sich nicht selber aufgeben wollte, eine Auswahl aus dem aufgeklärten Denken traf, dem er zu folgen gesonnen war. Das Staatsdenken der Aufklärer und der Aufgeklärte Absolutismus waren im Ergebnis zwei verschiedene Dinge « (S. 38); mit weniger negativer Beurteilung, aber in der Sache übereinstimmend: Duchhardt: Das Zeitalter des Absolutismus, S. 126.

${ }^{4}$ Schilfert: Deutschland von 1648 bis 1789, S. 168.

5 Duchhardt: Das Zeitalter des Absolutismus, S. 125.

6 Der an sich richtigen Feststellung, daß die Bedingungen einer Revolution im Deutschland des 18. Jahrhunderts fehlten, muß entgegengesetzt werden, daß es nicht um die objektive Unmöglichkeit des Umsturzes ging, sondern um die Wahrnehmung der Fürsten, die z.T. durch die durch das ganze Jahrhundert schwelenden 
gierte sich mit dem Absolutismus und stellte sich mit dem Anspruch zufrieden, sie setze das aufgeklärte Reformwerk in die Wirklichkeit um, aber damit täuschte sie sich und untermauerte den autoritären Staat für die Gegenwart und die Zukunft, als sie ihm ihre Befürwortung verlieh.

Die vorliegende Arbeit geht das Problem jedoch nicht von dieser theoretischen Seite an; sie beabsichtigt auch nicht, jene Untersuchung über Goethe und den aufgeklärten Absolutismus zu sein. Vielmehr will sie die oben zitierte Aussage Goethes von der historischen Seite überprüfen, indem sie die dunkle Seite der angeblich so aufgeklärten Regierung im Herzogtum Weimar beleuchtet und damit eine Art Vorstudie $\mathrm{zu}$ jener allgemeineren Untersuchung liefern will. Es geht hier um ein Schweigen, ja um ein Verschweigen in vieler Hinsicht. Daß Goethe nämlich Mitglied eines Geheimbunds war, der als Fernziel wenigstens nominell die Abschaffung von Monarchen und Staaten anstrebte, ist eine Tatsache, die von Goethe selbst verschwiegen und wegen Geheimhaltung der Quellen auch unter den meisten Spezialisten unbekannt blieb. Nach dem Wiederauftauchen dieser Quellen, die vor ihrem Verschwinden im Jahre 1936 nie eingehend ausgewertet worden waren, sind wir in der glücklichen Lage, die Tätigkeit dieser sIlluminaten in Weimar erforschen zu können. Daß sich speziell auch drei ungedruckte Goethe-Briefe (sowie auch einer von Herder und verschiedene Dokumente von Herzog Carl August) darunter befinden, ist für die GoetheForschung eigentlich kaum wichtiger als die zahlreichen Erörterungen über Goethes Beziehungen zu diesem Geheimbund in den Briefen anderer (die etwas ungewöhnliche Geschichte dieser Dokumente, der sSchwedenkiste, wird in Abschnitt 3.1 skizziert). Die auffallende Diskrepanz zwischen den angeblich radikalen politischen Zielen dieses Geheimbunds und den soeben erörterten konservativen politischen Anschauungen Goethes werden in der Untersuchung natürlich von Interesse sein. Für die vorliegende Fragestellung steht nämlich nicht Goethes Illuminatenmitgliedschaft als solche im Mittelpunkt - obwohl

Bauernunruhen geprägt war. Friedrich II. erklärte außerdem ausdrücklich, ein guter Fürst werde nie einen Aufruhr erleben; damit gab er zu verstehen, daß die Gefahr des Aufruhrs immer präsent war, und daß nur der sich aufgeklärt gebärdende Fürst der Aufklärung die kritische Spitze abbrechen könnte. Ingrid Mittenzwei resümiert: "Revolutionäre Erhebungen zu vermeiden, das war das eigentliche Ziel der Beschränkungen, die sich nach Friedrichs Meinung ein aufgeklärter Fürst auferlegen muß« (Mittenzwei: Über das Problem des aufgeklärten Absolutismus, S. 1168, das Friedrich-Zitat ebda.; ähnlich Schilfert: Deutschland von 1648 bis 1789, S. 171). 
auch diese als notwendige Voraussetzung dargestellt wird - sondern seine Teilnahme an Unterdrückung und Einschüchterung progressiver Kräfte in seiner Eigenschaft als leitender Beamter im Herzogtum Sachsen-Weimar; das Bindeglied zwischen beiden Tätigkeitsbereichen ist die These, daß Goethe und sein Herzog nicht aus Engagement dem Illuminatenorden beitraten, sondern zum Zweck der Überwachung. Gegenstand der Untersuchung ist die Angst vor konspirativer Verschwörung, die man besonders nach dem Anfang der Französischen Revolution hinter jeder geheimen Organisation der Intelligenz witterte, im weiteren Sinne der Argwohn gegen Intellektuelle überhaupt. Die geheimgehaltene eigene illuminatische Mitgliedschaft war für Goethe nur ein weiterer Aspekt seiner gleichfalls geheimgehaltenen Teilnahme an jener Überwachung und Einschüchterung der Intelligenz. Beide Bereiche seines Lebens verschwieg er.

Die folgenden Ausführungen haben jedoch nicht nur Goethes Biographie zum Gegenstand. Sie beleuchten auch nicht primär den Niederschlag dieser Thematik in Goethes Werken - er hat sie über Ansätze hinaus eben nicht in sein Repertoire von Themen aufgenommen, die er literarisch verarbeitet oder sonst zu einem Teil seiner "großen Konfession" gemacht hat; er versteckte dieses Kapitel seines Lebens sorgfältig bei der Konzeption seiner autobiographischen und fiktiven Schriften (abgesehen von einigen Anspielungen, die aber eher verdeckten als aufdeckten). Im folgenden geht es vielmehr um politische Vorgänge im Herzogtum Weimar, die eine geheime Schattenseite der Weimarer Klassik und einiger ihrer Hauptfiguren darstellen. Dies ist um so wichtiger, da die >Liberalität ‘ der Weimarer Regierung unter Herzog Carl August nicht nur ein politisches Alibi für Goethe liefert und zwar zu seiner eigenen Lebenszeit wie in der Forschung und der Öffentlichkeit bis heute -, sondern auch der deutschen Historiographie ein Beispiel für den angeblichen Erfolg des saufgeklärten ‘ Absolutismus geliefert hat: Carl August wird gern zusammen mit dem badischen Markgrafen Carl Friedrich als Repräsentant eines geglückten aufgeklärten Absolutismus gerühmt, nachdem die ehemals vornehmsten Vertreter, Friedrich II. (১der Große $\iota$ ) von Preußen, Joseph II. von Österreich und Catharina II. von Rußland, viel von ihrem Glanz verloren hatten. Inwieweit diese Legitimationsstrategie für die weitere Entwicklung der deutschen Geschichte verantwortlich gemacht werden kann - ob z.B. die in der Geschichtsforschung gestreifte Tatsache, daß sich im 20. Jahrhundert der Totalitarismus in ausgerechnet den Ländern entwickelte, die im 18. Jahrhundert den aufgeklärten Absolutismus kannten (Deutschland, Rußland, Italien, Spanien), aussagekräftig ist, ob etwa die 
Idee Martin Heideggers, den »Führer führen « zu wollen, auf altetablierte Vorstellungen des Fürstenmentors im aufgeklärten Absolutismus zurückgeht und damit die oft beschworene Nähe Buchenwalds zu Weimar zu mehr als einer geistreichen Pointe macht: Das alles muß hier im Hintergrund bleiben. Fest steht jedenfalls, daß der aufgeklärte Absolutismus auch in Weimar für die leider ewig junge Idee steht, daß alles Gute svon oben kommt und daß daher die Intelligenz ihre soziopolitischen Wirkungsmöglichkeiten in der Bindung an den starken Staat sehen sollte. Es kann hier auch nicht darum gehen, das Bild der Weimarer Liberalität grundlegend zu untersuchen, da hier nur ein Teilaspekt der Weimarer Machtverhältnisse dargestellt wird. Aber in der Anlehnung an die reaktionären Ideen der Verschwörungstheoretiker im Zeitalter der Französischen Revolution und noch davor sowie in der konsequenten Einschüchterung der öffentlichen Meinungsäußerung bzw. -bildung zeigt sich doch ein ganzes Stück der Weimarer Wirklichkeit, die sich hinter schönen (und manchmal sehr konservativen) dichterischen Werken verbirgt. Mit einer Analyse der Beteiligung Goethes an dieser Machtausübung ist natürlich über den globalen Wert seiner Dichtungen nichts ausgesagt - sie stehen hier sowieso am Rande -, sondern es geht um den Fall eines großen Schriftstellers, dessen politische Ansichten über das Vehikel seines Dichterruhms in die moderne Legitimierung eines starken Staates sozusagen hineingeschmuggelt wurden.

Diese Fragestellung läuft einem zentralen Anliegen der linken Literaturwissenschaft entgegen, zu der die vorliegende Untersuchung sich jedoch rechnet. Denn es geht seit eh und je darum, wichtige Schriftsteller für eine progressive Haltung zu reklamieren. Wenn dies sorgfältig durchgeführt wird, so hat es eine wichtige Funktion, aber allzuoft werden die dichterischen Werke und die historischen Gegebenheiten oberflächlich behandelt, um zum Ziel der progressiven Vereinnahmung eines politisch eher konservativen Schriftstellers zu gelangen. Das Resultat zeugt dann häufig von der Unsicherheit einer engagierten Literaturwissenschaft, der es nicht genügt, eine ansehnliche progressive Literaturtradition - einen Forster, Heine oder Brecht - sauf ihrer Seite zu wissen. Goethe ist das Paradebeispiel für dieses Verfahren, aus den oben dargelegten ideologischen Gründen. Die vorliegende Arbeit geht von der Voraussetzung aus, daß es wenig nützt, einen Schriftsteller für die eigene Position zu reklamieren, wenn die Oberflächlichkeit der Analyse leicht durchschaut werden kann. Viel wichtiger für die alltägliche Lebenspraxis ist es doch wohl, das kritische Instrumentarium dadurch zu schärfen, daß man die Vereinnahmung der literarischen Intelligenz durch Macht und andere soziopolitische Interessen zu erken- 
nen sucht. Es ist zu hoffen, daß dieser Ansatz die Einseitigkeiten der ideologiekritischen Literaturwissenschaft der vergangenen Jahrzehnte vermeiden und neuere theoretische Impulse in sich aufnehmen kann. Zentral für die vorliegende Untersuchung war die theoretische Frage, ob angesichts der darzustellenden Machtverhältnisse von einer autonomen Subjektbildung gesprochen werden kann, wie sie gerade von der Weimarer Klassik postuliert wird. Eine andere Leitfrage war das Problem der Opposition unter den Bedingungen einer Vereinnahmung der Intelligenz durch den autoritären Staat. Schließlich muß nach der potentiellen >Dialektik der Aufklärung im Illuminatenorden gefragt werden; war es unter den gegebenen Bedingungen nötig, daß die geheime Organisation der Intelligenz zu geradezu despotischen Maßnahmen griff, daß sie einen instrumental gesteuerten »Retortenmenschen der Aufklärung « ${ }^{7}$ schaffen mußte, um ihre Zwecke durchzusetzen?

Diese Arbeit versteht sich als Plädoyer nicht nur für eine historische und ideologiekritische Verankerung der Literatur- und Kulturgeschichte, sondern auch für eine erneute Beschäftigung mit vernachlässigten Quellen, gedruckten wie ungedruckten. Daß im vorliegenden Fall eine wichtige Quellensammlung wieder aufgetaucht ist, ist freilich ein glücklicher Zufall, der sich heute immer seltener ereignet. Aber ein großer Teil der Quellen für diese Untersuchung entstammt eben nicht jener `Schwedenkiste`, sondern anderen, schon längst bekannten archivalischen Beständen sowie verstreut und versteckt gedruckten Quellen. ${ }^{8}$ Es trifft wohl zu, daß fast alle Schriften und Briefe einiger der bedeutendsten Schriftsteller in der deutschen Literaturgeschichte publiziert oder wenigstens bekannt sind. Aber im historischen Kontext ihres Wirkens - nicht nur in der Wirkung bzw. Rezeption ihrer Schriften, sondern auch im politischen Umfeld ihres Lebens - liegt in vielen Fällen ein braches Feld. Daß gerade mit Bezug auf den Weimarer Herzog, Goethes engen Freund und politischen Kollegen, eine Menge unveröffentlichter Quellen über die Behandlung der Professorenschaft an der Universität Jena benutzt werden konnte, wo die romantische Bewegung ih-

${ }^{7}$ So Voges: Aufklärung und Geheimnis, S. 103, der auch die Frage nach der >Dialektik der Aufklärung ‘ aufwirft.

${ }^{8} \mathrm{Zu}$ erwähnen sind besonders ältere Quellen wie Diezmann: Aus Weimars Glanzzeit (vgl. Kap. 5.3); außerdem konnten in einer Reihe von Fällen Briefe des Herzogs Carl August benutzt werden, von denen nur Auszüge in seinem >Politischen Briefwechsel (CAPB) veröffentlicht worden waren; gerade die ausgelassenen Stellen mancher Briefe interessierten am meisten für die vorliegende Fragestellung (vgl. wiederum alle Teile von Kap. 5). 
ren Ursprung hatte, sollte bedenklich stimmen gegenüber der allgemein akzeptierten Annahme, daß Archivarbeit nur über neu zu entdeckende Autoren ausgeführt werden könne.

Für entgegenkommende Hilfeleistung bin ich folgenden Archiven und Personen zu besonderem Dank verpflichtet: der Königlichen Bibliothek, Kopenhagen; dem Staatsarchiv Weimar; dem Goethe- und Schiller-Archiv der Nationalen Forschungs- und Gedenkstätten der klassischen deutschen Literatur in Weimar (besonders Günter Arnold und Gerhard Schmid); der Forschungsbibliothek Gotha und der Leiterin der Handschriftenabteilung, Maria Mitscherling. Auch dem Personal der Staats- und Universitätsbibliotheken Hamburg, Göttingen und München, sowie der Herzog August Bibliothek Wolfenbüttel und der Zentralbibliothek der deutschen Klassik in Weimar ist zu danken. Besonderen Dank möchte ich den Archivarinnen des Zentralen Staatsarchivs der DDR, Dienststelle Merseburg, aussprechen; Archivrätin Renate Endler half mir mit ihrem Fachwissen über den Bestand der sSchwedenkiste und förderte meine Forschungen auf sehr kollegiale Weise. Folgende studentische Hilfskräfte unterstützten mich in verschiedenen Stadien der Arbeit: Samuel Dunlap, Kay Henschel, Linda von Hoene, Wayne Miller und Gail Wise (Berkeley); Angelika Bartoldus (Hamburg); Ines Engelmann, Anke Nothnagel und Anke Pätsch (Leipzig/Merseburg); und besonders Christina von Hodenberg (München/Merseburg). Ein Forschungsaufenthalt in der Bundesrespublik und die Archivarbeit in der DDR im Jahre 1989/90 wurden durch die Unterstützung des Committee on Research, University of California at Berkeley, ermöglicht. Meine Kollegen in Berkeley haben die Arbeit auf vielfältige Weise gefördert.

Frühere Fassungen von Kapitel 5.3 bzw. Kapitel 4 und 3.3 erschienen in englischer Sprache in den Zeitschriften Euphorion und Goethe Yearbook (1989). Die dort veröffentlichten Ergebnisse wurden jedoch auf Grund der archivalischen Arbeit überprüft und grundlegend revidiert bzw. beträchtlich erweitert. Die Arbeit wurde (von einigen Nachträgen abgesehen) im August 1990 abgeschlossen. 